\title{
OPTICAL AND ULTRASONIC INVESTIGATIONS OF PHASE TRANSITIONS IN FERROELECTRIC $\mathrm{RbHSeO}_{4}$ SINGLE CRYSTAL
}

\author{
S.A. Sveleba, M.I. Bublik, I.I. Polovinko, A.V. Kityk, V.P. Sopronuk \\ Lviv State Universily, Universitetska 1, 290005, Lviv, Ukraine
}

\author{
AND Z. CZAPla
}

Institute of Experimental Physics, University of Wrocław

Cybulskiego 36, 50-205 Wrocław, Poland

(Received May 4, 1992)

\begin{abstract}
Birefringence and ultrasonic measurements were done for ferroelectric $\mathrm{RbHSeO}_{4}$ crystal. Influences of both stresses $\left(\sigma_{i}\right)$ and pressure on the properties and phase transition were studied as well. The observed dependence of $\delta\left(\Delta n_{\mathrm{b}}\right)=f\left(\sigma_{5}\right)$ shows hysteresis loop characteristic of ferroelectric phase whereas the dependence of $\delta\left(\Delta n_{c}\right)=f\left(\sigma_{6}\right)$ shows hysteresis loop characteristic of ferroelastic phase. Birefringence measurements prove the phase transition to ferroelectric phase at $373 \mathrm{~K}$. Additionally, the phase transition was found at $398 \mathrm{~K}$. The new phase may be regarded as an incommensurate one. On the grounds of the stresses infuence the phase diagram is constructed in coordinates $T, \sigma_{5}$. The linear decrease in $T_{c}$ with increase in pressure is observed in ultrasonic studies and it is similar to the results obtained in DSC measurements.
\end{abstract}

PACS numbers: 64.70.Kb, 77.20.+y, 05.70.Fh

\section{Introduction}

Rubidium hydrogen selenate $\mathrm{RbIISeO}_{4}$ is known as hydrogen-bonded ferroelectric crystal with $T_{\mathfrak{c}} \approx 371 \mathrm{~K}$ [1]. In this crystal, simultaneously ferroelectric and ferroelastic domain structure with the walls parallel to (001) planc exists below $T_{\mathrm{c}}$. The observed domain structure pattern may be changed by application of electric field along the $b$-axis or mechanical stress $\left(\sigma_{5}\right)$ to the $b-45^{\circ}$ cut samples [2]. Occasionally, ferroelastic domain structure with the walls parallel to the plane (100) exists both below and above $T_{c}$. This kind of domain structure may be changed by the mechanical stress $\left(\sigma_{6}\right)$ applied to the $c-45^{\circ}$ cut samples. The aim of our work 
is to obtain some additional information related to the phase transitions diagram, domain structure and piezooptic properties on the grounds of birefringence and ultrasonic measurements. The birefringence measurements were also used to check on the possibility of incommensurate phase existence in $\mathrm{RbHSeO}_{4}$ crystal.

\section{Experimental}

Single crystal of $\mathrm{RbHSeO}_{4}$ of optical quality were grown by slow evaporation of an aqueous solution containing stoichiometric quantities of $\mathrm{Rb}_{2} \mathrm{SeO}_{4}$ and $\mathrm{H}_{2} \mathrm{SeO}_{4}$ at the temperature of $301 \mathrm{~K}$. The temperature dependences of birefringence and piezooptic coeflicients were measured by Senormonth's method $(\lambda=$ $633 \mathrm{~nm})$ using Faraday modulator cell. The accuracy of $\delta(\Delta n)$ measurements is equal to $10^{-7}$. The temperature was kept and measured with the accuracy of $0.01 \mathrm{~K}$. The accuracy of piezooptic coefficients is estimated as equal to about $10 \%$. The shear ultrasonic wave (USW) velocities $v_{4}(q\|b, E\| b)$ and $v_{5}(q\|c, E\| a)$, where $q$ is a USW wave vector, $\boldsymbol{E}$ - polarization, were measured by the pulse-echo overlap method [3] at the frequency $f=10 \mathrm{MIIz}$ with the accuracy of $10^{-4}-10^{-5}$. Acoustic investigations under hydrostatic pressure were performed in the range of 0.1 to $500 \mathrm{MPa}$ and $300-400 \mathrm{~K}$ using a high-pressure camera.

\section{Results and conclusións}

An influence of internal stresses $\sigma_{i}$ on the birefringence of $\mathrm{RbIISeO}_{4}$ crystal is shown in Figs. 1-5.

The mechanical stress $\sigma_{5}$ applied to the sample at room tempcrature gives a decrease in $\delta\left(\Delta n_{\mathrm{b}}\right)$ for the stresses $\sigma_{5}<0.70 \times 10^{6} \mathrm{~N} / \mathrm{m}^{2}$. For the stresses

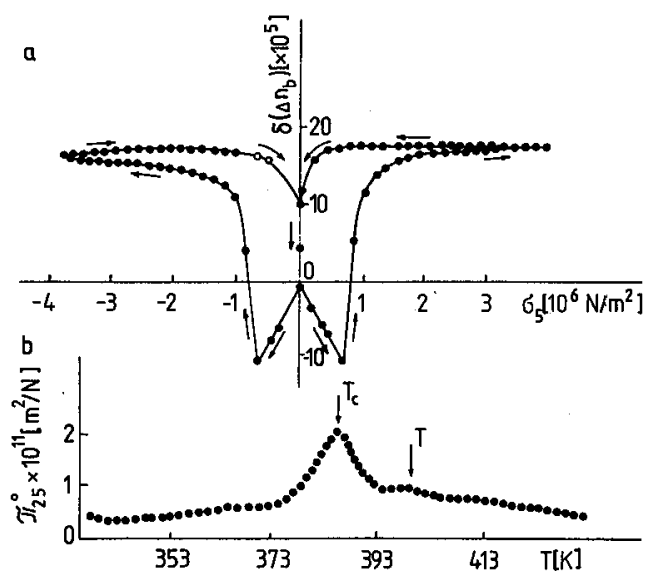

Fig. 1. (a) Dependence of $\delta\left(\Delta n_{\mathrm{b}}\right)$ versus $\sigma_{5}$ at room temperature; (b) temperature dependence of piezooptic coefficient $\Pi_{25}^{0}$; (a) and (b) for $\mathrm{RbHSeO}_{4}$ crystal.

$\sigma_{5}>0.70 \times 10^{6} \mathrm{~N} / \mathrm{m}^{2}$ a sharp increase in $\delta\left(\Delta n_{\mathrm{b}}\right)$ is observed and then its saturated value is reached. When the mechanical stress is removed, $\delta\left(\Delta n_{\mathrm{b}}\right)$ slightly 


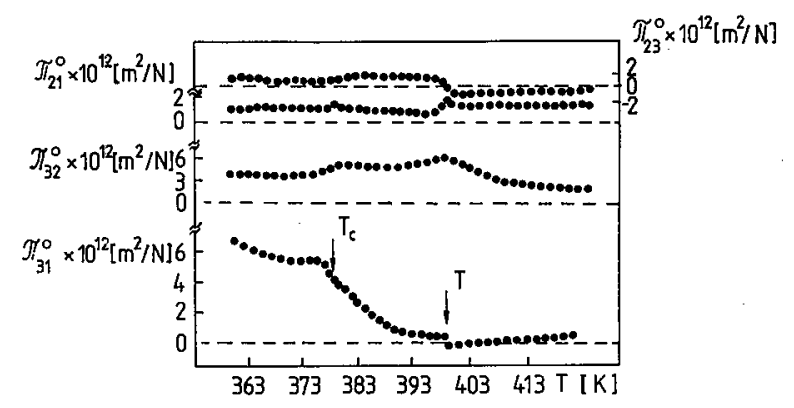

Fig. 2. Temperature dependences of some piezooptic coefficients $\Pi_{i k}^{0}$ for $\mathrm{RbHSeO}_{4}$ crystal.

decreases and after that the relaxation process is observed and nearly initial value for $\delta\left(\Delta n_{\mathrm{b}}\right)$ is reached in some time (see the right side of Fig. 1a). If the sample is turned by $90^{\circ}$ around the $b$-direction, the observed dependence of $\delta\left(\Delta n_{\mathrm{b}}\right)$ on $\sigma_{5}$ is shown in the left side of Fig. 1a. The dependence of $\delta\left(\Delta n_{\mathrm{b}}\right)=f\left(\sigma_{5}\right)$ (hysteresis loop) presented in Fig. 1a is similar to the dependence of $\delta(\Delta n)$ on $E$ observed in ferroelectric phases. $\mathrm{RbHSeO}_{4}$ crystal is simultaneously ferroelectric and ferroelastic and mechanical stress influence also gives histeresis loop. At room temperature the value of coercive stress $\left(\sigma_{5}\right)_{\mathrm{c}}$ for $\mathrm{RbIISeO}_{4}$ is equal to about $0.75 \times 10^{6} \mathrm{~N} / \mathrm{m}^{2}$. Figure 1b also shows the temperature dependence of piezooptic coefficient $\Pi_{25}^{0}$ obtained for $\sigma_{5}>\left(\sigma_{5}\right)_{c}$. As can be seen in Fig. $1 \mathrm{~b}$, a big anomaly at $T=385 \mathrm{~K}$ and a kink at $T_{\mathrm{i}} \approx 398 \mathrm{~K}$ on the curve $\Pi_{25}^{0}=f(T)$ are found. The observed anomaly at $385 \mathrm{~K}$ corresponds to the phase transition at $T_{\mathrm{c}}=373 \mathrm{~K}$ (the value obtained in this work) shifted to higher temperature by application of external stress.

To explain the observed kink at $T_{\mathrm{i}}$ the dependences of $\delta\left(\Delta n_{i}\right)=f(T)$ and $\Pi_{i k}^{0}=f(T)$ were measured. According to the dependences of $\delta\left(\Delta n_{\mathrm{b}}\right)=f(T)$ and $\delta\left(\Delta n_{\mathrm{c}}\right)=f(T)$ the crystal undergoes the first- and second-order phase transitions at $T_{\mathrm{c}}$ and $T_{\mathrm{i}}$, respectively. The first-order phase transition had been found earlier in dielectric [1] and birefringence measurements [4]. Temperature dependences of $\Pi_{i k}^{0}$ are shown in Fig. 2. The anomalies of $\Pi_{21}^{0}, \Pi_{23}^{0}$ and $\Pi_{31}^{0}$ were found at $T_{\mathrm{c}}$ and $T_{\mathrm{i}}$. They also give evidences for the phase transitions at these temperatures.

Temperature dependence of $\delta\left(\Delta n_{\mathrm{b}}\right)$ at various values of $\sigma_{5}$ is shown in Fig. 3. As can be seen in Fig. 3 the difference $T_{\mathrm{i}}-T_{\mathrm{c}}$ decreases with increase in $\sigma_{5}$ whereas one diffused phase transition for $\sigma_{5} \geq 3.6 \times 10^{6} \mathrm{~N} / \mathrm{m}^{2}$ is observed. For $\sigma_{5}>5 \times 10^{6} \mathrm{~N} / \mathrm{m}^{2}$ this transition is hardly distinguished. The similar shift of $T_{\mathrm{c}}$ versus $\sigma_{5}$ was observed in dielectric measurements [5].

The presented above measurements allowed us to construct the phase diagram in coordinates $T$ and $\sigma_{5}$ which is shown in Fig. 4. To explain the obtained diagram one can assume that the phase existing in the temperature range $T_{\mathrm{i}}-T_{\mathrm{c}}$ is an incommensurate one. Thus, $T_{\mathrm{c}}$ is the temperature of phase transition to the ferroelectric phase and $T_{\mathrm{i}}$ is the phase transition temperature to the incommensurate one. The incommensurate phase existence was proved in an isomorphous ammonium hydrogen selenate $\left(\mathrm{NH}_{4} \mathrm{ISeO}_{4}\right)$ crystal [6-8], as it is shown in Fig. 4 . 

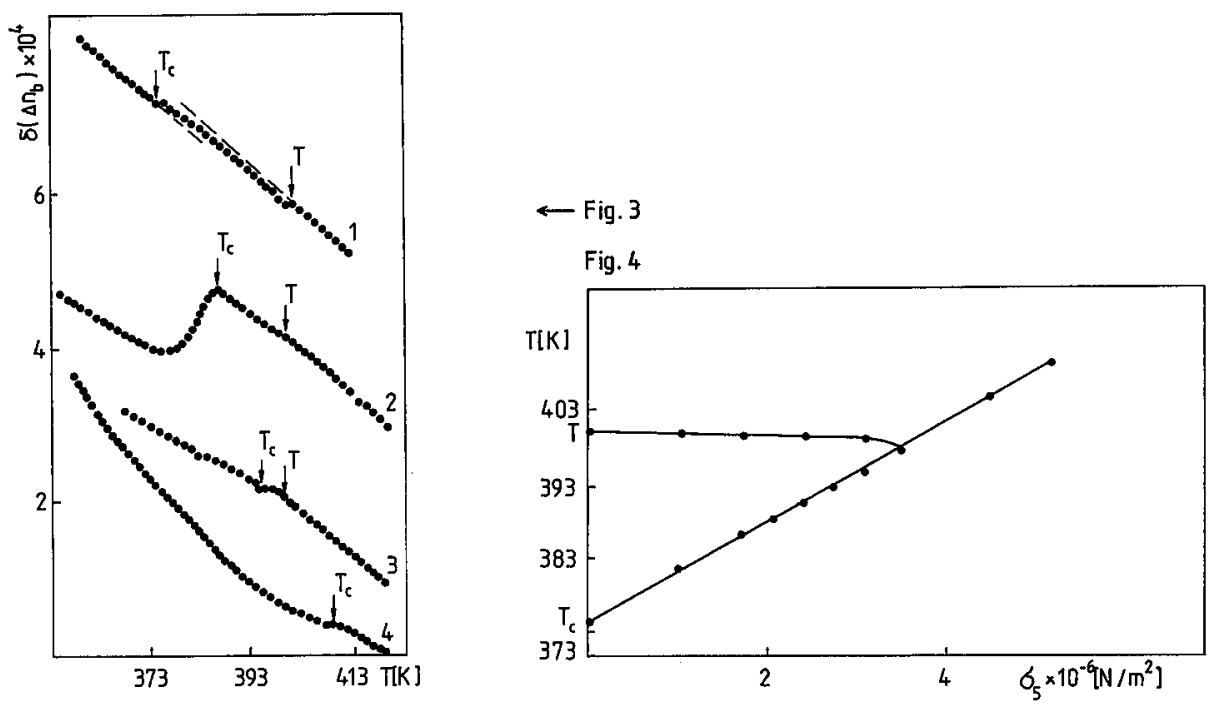

Fig. 3. Temperature dependences of $\delta\left(\Delta n_{\mathrm{b}}\right)$ for $\mathrm{RbHSeO}_{4}$ crystals at various $\sigma_{5}$ $\left[\times 10^{-6} \mathrm{~N} / \mathrm{m}\right]: 1-0,2-1.7,3-3.1,4-5$.

Fig. 4. Phase diagram for $\mathrm{RbHSeO}_{4}$ crystal; dependences of phase transition temperatures versus $\sigma_{5}$.

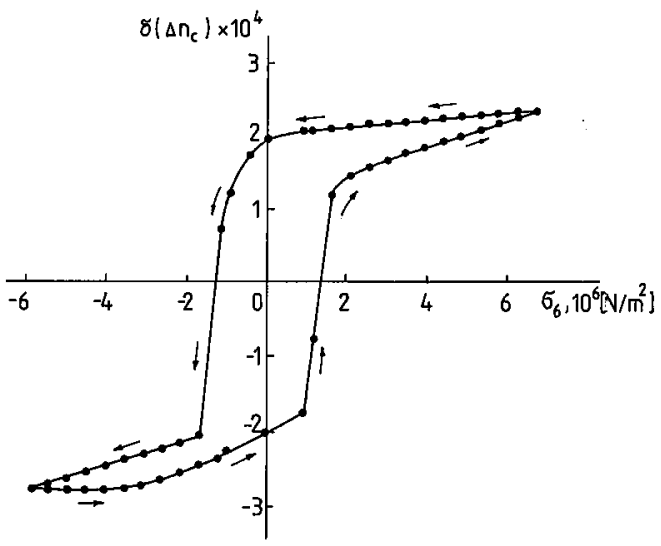

Fig. 5. Dependence of $\delta\left(\Delta n_{c}\right)$ versus $\sigma_{6}$ for $\mathrm{RbHSeO}_{4}$ crystal.

The dependence of $T_{\mathrm{i}}$ on $\sigma_{5}$ is linear and diminishes very slightly with increase in $\sigma_{5}$. The dependence of $T_{\mathrm{c}}$ on $\sigma_{5}$ is also linear up to $5 \times 10^{6} \mathrm{~N} / \mathrm{m}^{2}$ with the rate of $\Delta T_{\mathrm{c}} / \Delta \sigma_{5} \approx 7.5 \times 10^{6} \mathrm{~K} \cdot \mathrm{m}^{2} / \mathrm{N}$. The dependence of $T_{\mathrm{c}}$ versus $\sigma_{5}$ is characteristic of proper ferroelectrics with the first-order phase transition without incommensurate phase. Both dependences (straight lines) meet at about $3.6 \times 10^{6} \mathrm{~N} / \mathrm{m}^{2}$ and this value is a limit of the incommensurate phase existence. Thus, the phase diagram 
contains triple point in which the incommensurate phase disappears. For stresses higher than $3.6 \times 10^{6} \mathrm{~N} / \mathrm{m}^{2}$ we deal with the direct transition from ferroelectric to paraelectric phase. For stresses higher than $5 \times 10^{6} \mathrm{~N} / \mathrm{m}^{2}$ the ferroelectric commensurate phase and the normal phase are indistinguishable.

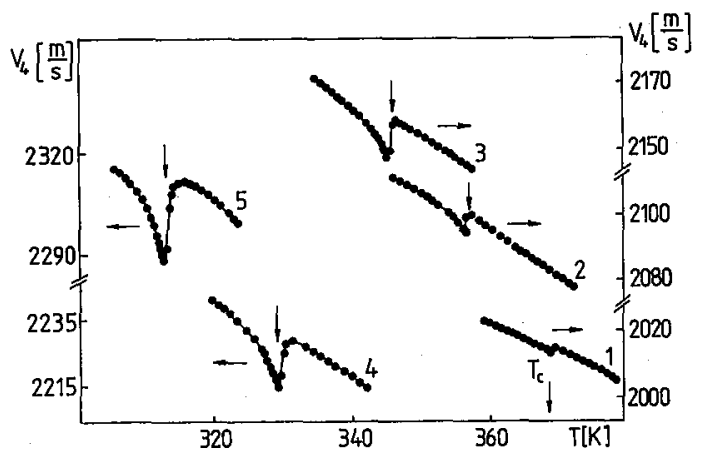

Fig. 6. Temperature dependence of the shear velocity $v_{4}$ for various $p$ [MPa] $\left(\mathrm{RbHSeO}_{4}\right.$ crystal): $1-0.1,2-95,3-185,4-325,5-475$.

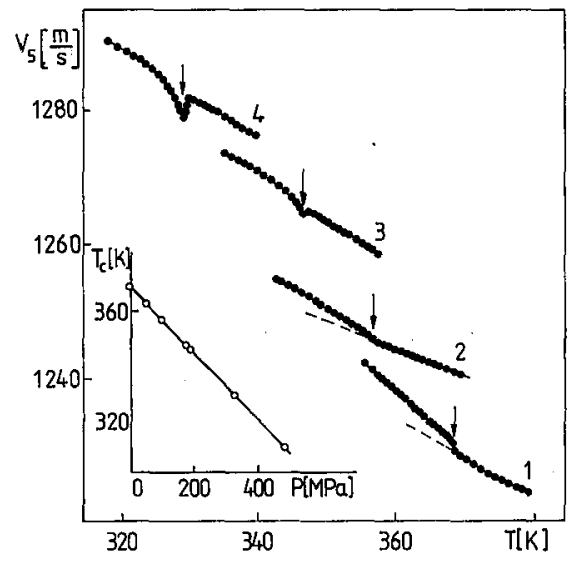

Fig. 7. Temperature dependence of the shear velocity $v_{5}$ for various $p[\mathrm{MPa}]\left(\mathrm{RbHSeO}_{4}\right.$ crystal): $1-0.1,2-95,3-185,4-325$.

In connection with the existence of ferroelastic domain structure observed along the $c$-axis the dependence of $\delta\left(\Delta n_{c}\right)$ on mechanical stress $\sigma_{6}$ was studied. The hysteresis loop $\delta\left(\Delta n_{c}\right)=\int\left(\sigma_{6}\right)$ obtained at room temperature phase is shown in Fig. 5. As can be seen in Fig. 5 the shape of the observed hysteresis loop is characteristic of ferroelastic crystals. The value of the coercive mechanical stress $\left(\sigma_{6}\right)_{\mathrm{c}}$ is equal to about $1.3 \times 10^{6} \mathrm{~N} / \mathrm{m}^{2}$ at room temperature and the hysteresis loop may be regarded as symmetrical in respect to both coordinates.

The results of ultrasonic measurcments are presented in Figs. 6 and 7. 
Anomalies of shear USW velocities $v_{4}$ and $v_{5}$ were observed at $T_{\mathrm{c}}$. At the pressure $p=0.1 \mathrm{MPa}$ a small decrease in $v_{4}$ (Fig. 6) and a small jump of $v_{5}$ (Fig. 7) can be seen at the phase transition temperature $T_{\mathrm{c}}$. Under applied hydrostatic pressure the phase transition temperature $T_{\mathrm{c}}$ shifts to lower temperatures with the rate equal to $-0.126 \mathrm{~K} / \mathrm{MPa}(-12.6 \mathrm{~K} / \mathrm{kbar})$. Simultaneously, an increase in the $v_{4}$ jump at $T=T_{\mathrm{c}}$ is observed with increase in the value of applied pressure. In the case of $v_{5}$ the change of the anomaly character takes place (see Fig. 7). The shift of $T_{\mathfrak{c}}$ is characteristic of hydrogen-bonded ferroelectrics (see the insert in Fig. 7) and is in good agreement to that observed from DSC measurements [9].

On the grounds of presented above investigations one can state that:

1. two different forms of $\delta\left(\Delta n_{i}\right)=f\left(\sigma_{i}\right)$ dependences are observed in $\mathrm{RbIISeO}_{4}$ crystal: the dependence of $\delta\left(\Delta n_{\mathrm{b}}\right)=\int\left(\sigma_{5}\right)$ is characteristic of ferroelectric phase and the dependence of $\delta\left(\Delta n_{\mathrm{c}}\right)=f\left(\sigma_{6}\right)$ is characteristic of ferroelastic one;

2 . the existence of incommensurate phase is proposed and the phase diagram in coordinates "temperature" $(T)$-stress $\left(\sigma_{5}\right)$ was constructed;

3 . the influence of pressure on the phase transition at $T_{\mathrm{c}}$ characteristic of hydrogen-bonded crystals was confirmed.

This work was partially supported by Grant Nr 201289101.

\section{References}

[1] Z. Czapla, T. Lis, L. Sobczyk, J. Mróz, R. Poprawski, Ferroelectrics 26, 771 (1980).

[2] T. Tsukamoto, M. Komukac, S. Suzuki, II. Futama, M. Makita, J. Phys. Soc. Jpn. 52, 3966 (1983).

[3] E.P. Papadakiz, J. Acoust. Soc. Am. 42, 1045 (1967).

[4] A. Waśkowska, A.M. Glazer, J. Phys. C, Solid State Phys. 19, 6221 (1992).

[5] R. Styrkowiec, private information.

[6] H. Pykacz, Solid Slale Commun. 66, 567 (1988).

[7] J. Kroupa, J. Fousek, Z. Czapla, Ferroelectrics 79, 291 (1988).

[8] F. Denoyer, A. Rozycki, K. Parliński, M. More, Phys. Rev. B 39, 405 (1989).

[9] B. Baranowski, M. Friesel, A. Lunden, Z. Nalurforsch. A 41, 981 (1986). 